\title{
Application of a hybrid approach to the design of anchored wire meshes on steep slopes
}

\author{
A Galli Politecnico di Milano, Italy \\ M Deana Officine Maccaferri SpA, Italy \\ N Mazzon Maccaferri Innovation Center, Italy
}

\begin{abstract}
Anchored steel meshes are commonly employed stabilising systems for potentially unstable granular layers (e.g. soil or highly fragmented weak rock) on slopes against translational failure mechanisms. They are usually conceived as passive stabilising intervention (i.e. their stabilising action is provided only upon the activation of a given displacement field into the unstable layer). As a consequence, standard design methods, generally based on ultimate limit state (ULS) approaches, can potentially be largely unsafe, since they assume the full mobilisation of both the ultimate soil resistance and the ultimate tensile force in the steel mesh independently of the displacement amplitude of the unstable layer. In this paper a design procedure, based on so called 'hybrid' approaches, is described with specific reference to relatively steep slopes. The key concept is the use of a suitable characteristic curve, describing the mechanical response of the entire system (steel plate, steel mesh, soil layer) as a function of the displacement amplitude, thus providing a safe and consistent estimation of the mobilised stabilising action for the required safety level.
\end{abstract}

Keywords: anchored steel meshes, design procedure, hybrid methods

\section{Introduction}

Despite the large amount of theoretical, numerical and experimental works developed in recent decades, design approaches and dimensioning criteria for slope stabilisation interventions are still a challenging issue for the engineers, since they are intrinsically characterised by high degrees of complexity. These are mainly due to nonlinearities and irreversibilities in soil mechanical behaviour, uncertainties in materials and driving load characterisations (especially when environmental loads are considered), and complex and multiscale interaction between the soil and the stabilising structure. This is particularly true in the case of anchored steel meshes installed upon unstable layers, when the stabilising action is governed by the complex interaction among all the components of the stabilising systems (steel mesh, anchored plates, unstable soil). For passive intervention, moreover, stabilising actions are triggered uniquely by the activation of a sliding process into the unstable layer. In the engineering practice, standard design approaches for these systems are usually based on ultimate limit state (ULS) methods, implicitly assuming the full mobilisation of the ultimate strength of both the soil and mesh, independently of the slope movement. As a consequence, ULS approaches rely only on an 'asymptotical' condition that can potentially never be reached in practice.

In the framework of ULS methods, however, current design standards usually introduce partial safety factors reducing the characteristic strength values. This choice, although implicitly, introduces then a limitation on the strain (and, more in general, displacement) pattern associated to the design values of the mobilised strength values. However, no clear stress-strain relationship is introduced, thus making it impossible to adopt a rational design approach, based on performance criteria.

Taking advantage of some recent results on sustainable design methods for slope stabilising interventions (Italian Ministry of Education, University and Research 2011), the present paper adopts the so called hybrid method to propose an innovative design procedure for stabilising steel meshes. In particular, in the following, an extension of the recent work discussed in Galli \& Deana (2020) for steep slopes is presented. The key 
concept is the use of a suitable characteristic function (or characteristic curve) to consistently describe the relationship between the contact pressures developed at the soil-mesh interface as a function of the underlying slope movement (i.e. the so called 'far field' displacement). The global stability of the slope can then be obtained by means of the usual limit equilibrium methods (LEM), where the stabilising action considers a realistic exercise condition for the mesh. In other words, hybrid methods combine a ULS approach for the slope stability assessment together with a serviceability limit state (SLS) approach for describing the soil-structure interaction. It is worth noting that hybrid methods cannot be interpreted as a simplified method to foresee the expected onsite soil profile displacements, since they do not describe the actual time evolution of the slope displacement field. They can only be considered as a simplified and rational way to compare different alternative design options, always being sure to get a safe and consistent result.

The innovative aspect of the present work is to introduce into a traditional two-block LEM (developed to assess the stability of a slope) a displacement-based estimation of the stabilising force, based on the concept of characteristic curves. This conceptually corresponds to the force-displacement curve resulting from traditional punching tests against soil, so that the curve can then be employed not only in order to study the ultimate strength of the stabilising system, but also to estimate the local displacement corresponding to the value of the mobilised stabilising force. For general applications, the specific characteristic curve to be adopted can be derived by developing a suitable soil-structure interaction model, based on the definition of the generalised stress and strain variables. This point being out of the scope of the present work (for the sake of brevity a detailed description of this upscaling process is omitted). An example of the application of the aforementioned hybrid method will be discussed by adopting as characteristic curve the results of an experimental punching test.

\section{Two-block approach}

With reference to the simplified slope geometry shown in Figure 1a, where several possible translational failure mechanisms are reported, a schematic two-block limit equilibrium analysis is adopted (Figure 1b). The upper block represents the unstable soil layer, whose sliding movement along the inclined bedrock is represented by a displacement vector $U$; the lower triangular block represents the toe condition for the failure mechanism, providing the resistant stabilising action against the sliding of the upper block. If an anchored mesh is installed along the slope, its stabilising action can be modelled as a distributed pressure $q$ along the 'active' zone of the failure mechanism (i.e. the length $L_{a}$ of the toe mechanism characterised by a normal displacement component $U_{N}$ with respect to the slope inclination $\alpha$ ). By imposing the equilibrium of the two blocks along both the directions normal and tangential to the slope, a direct relation between the Factor of Safety $F_{S}$ of the failure mechanism and the applied pressure $q$ can be found, once the length $L_{a}$ is assigned. Similarly to what it is generally done in LEM codes, the length $L_{a}$ is obtained by an optimising process: for a prescribed value of $F_{S}$, the resulting value of $q$ is maximised with respect to the angle $\beta$, changing the position of point $P^{\prime \prime}$ (Figure 1c).

With respect to what was recently proposed in Galli \& Deana (2020), where the definition of the toe block was based on the assumption of a local passive failure condition of the soil and its local displacement field was assumed to be perfectly horizontal, the aforementioned two-block approach is more suitable for steep slopes. In these latter cases, in fact, no displacement direction is a priori assumed for the toe block, but it is the result of an optimisation procedure, as briefly described here previously. The authors want, however, to clearly highlight that only a wise comparison between the two methods can give meaningful design indications. 


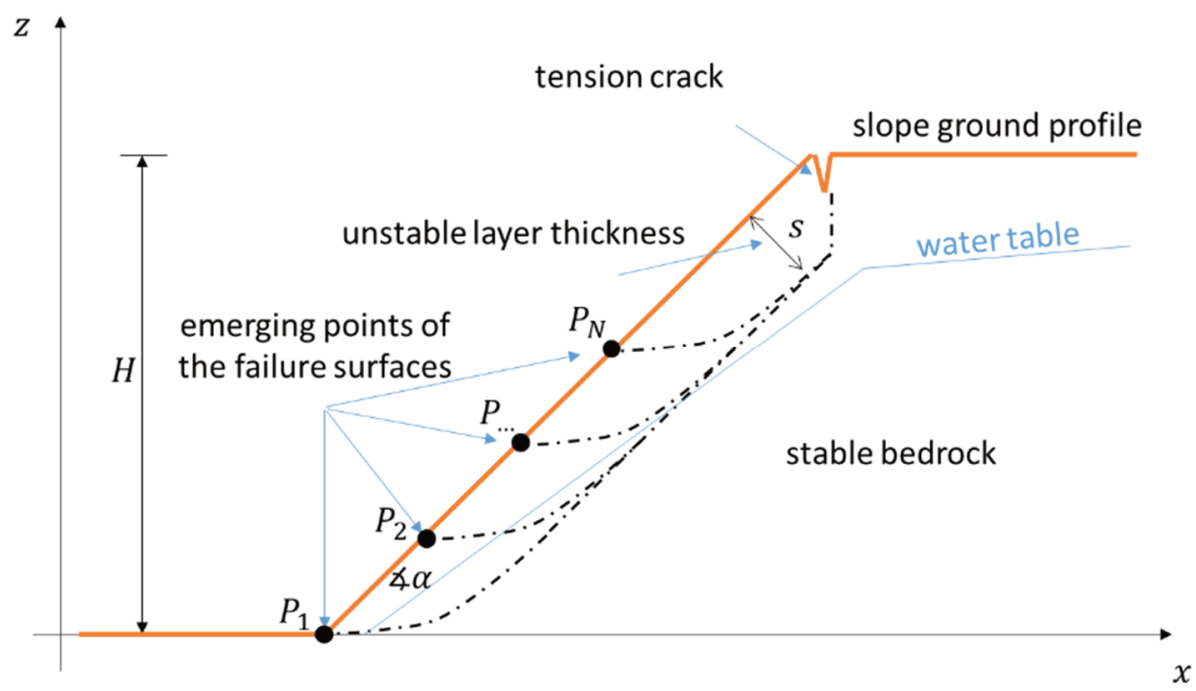

(a)

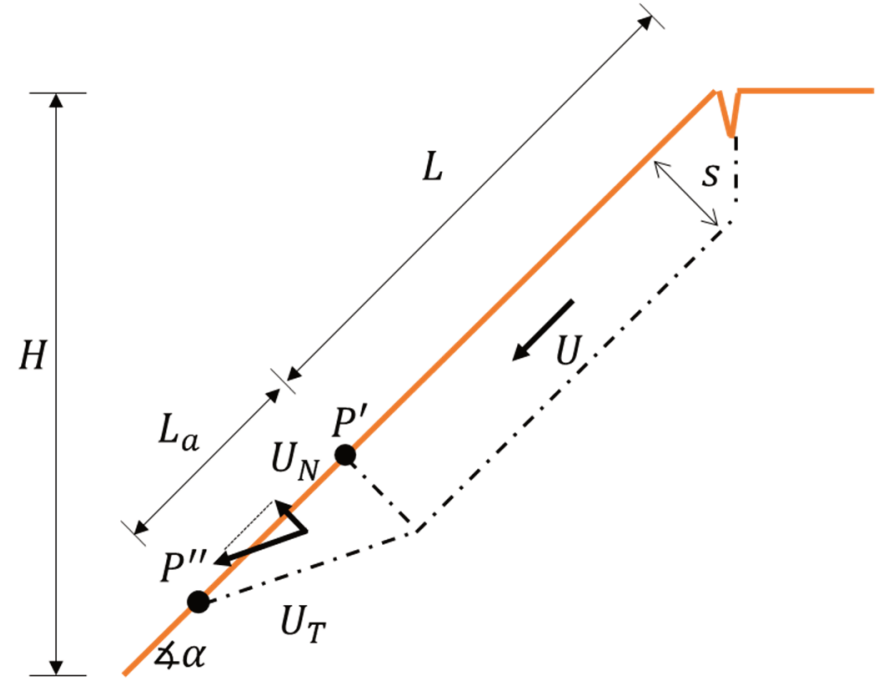

(b)

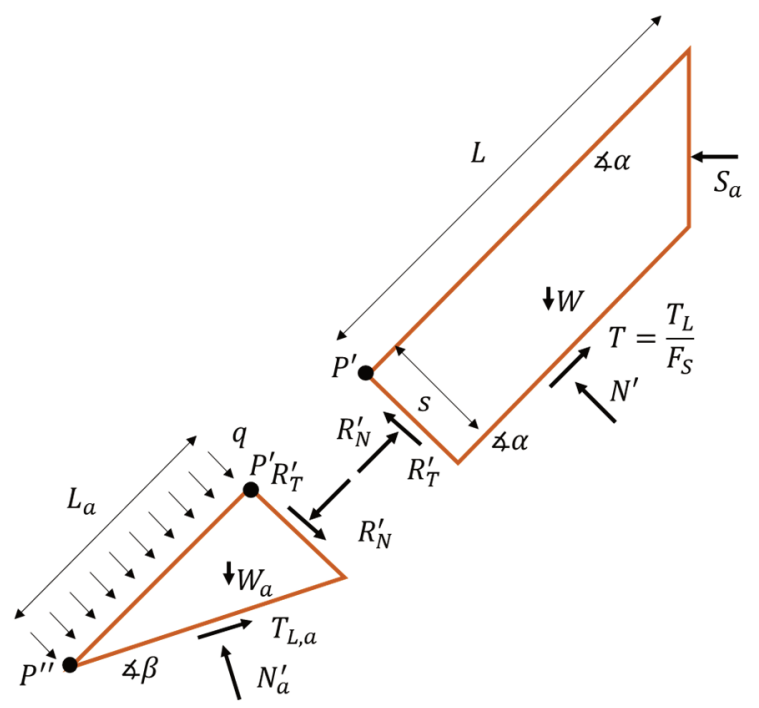

(c)

Figure 1 Schematic view of an unstable layer on a sloping ground: (a) General approach; (b) Failure mechanism; (c) Acting forces 


\section{The characteristic curve}

The key concept of the proposed hybrid method is to introduce a convenient relationship between the stabilising force (or, equivalently, its corresponding distributed pressure $q$ ) and the local normal displacement $U_{N}$ of the toe block. This relationship represents a sort of 'characteristic curve' of the system, providing an 'upscaled' description of the mechanical interaction between the soil, the steel mesh, the steel plate and the anchors from the local scale of the representative soil element volume (REV) to the macroscopic scale of the variables $q$ and $U_{N}$. As pointed out in many recent works concerning even other types of stabilising structures (see e.g. Galli \& di Prisco 2013; Galli et al. 2017), characteristic curves inherently consider both the mechanical properties of the materials (i.e. in this case the soil and the steel mesh) and the geometrical dimension of the system (i.e. the steel plate dimensions, the ground anchor spacing, unstable layer thickness, $s$ in Figure 1).

Accurate calibration of the characteristic curve is of course the crucial point in this method, and it can be obtained either by performing experimental punching tests against soil, or, more frequently, by running ad hoc 3D numerical simulations specifically conceived for the case under consideration. Boschi et al. (2019) have recently proposed 3D finite element method (FEM) numerical analyses investigating this point in further details, in particular highlighting the additional confining effect provided by the steel mesh all around the steel plate, which induces an increase of the bearing capacity of the plate against the local punching into the underlying soil. This effect, coupled with the membrane behaviour of the steel mesh, typically induce a remarkable stiffening effect on the characteristic curve. Experimental evidences of such behaviour are, for example, presented in Figure 2, were the results of some experimental punching test of anchored steel mesh against soil are shown (Figure 2a, modified from di Prisco et al. 2010). In Figure 2b, $F$ is the total normal force and $U$ is the normal plate displacement in punching test against soil, and a comparison is proposed between the behaviour of the system when a single plate without steel mesh or a plate+net are considered. In the following, consistently with what was already done in Galli \& Deana (2020), the experimental characteristic curve shown in Figure $2 \mathrm{c}$ is adopted, corresponding to a punching test run on a steel mesh characterised by a tensile stiffness of about $700 \mathrm{kN} / \mathrm{m}$ against a soil with geotechnical properties similar to those listed in Table 1 (further details are omitted for the sake of brevity).

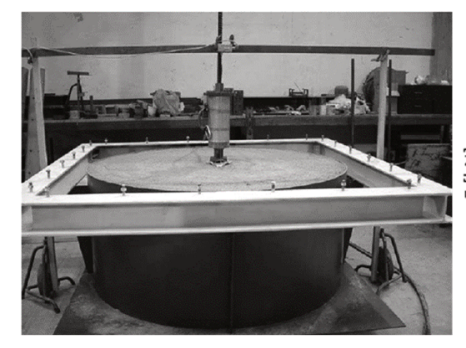

(a)

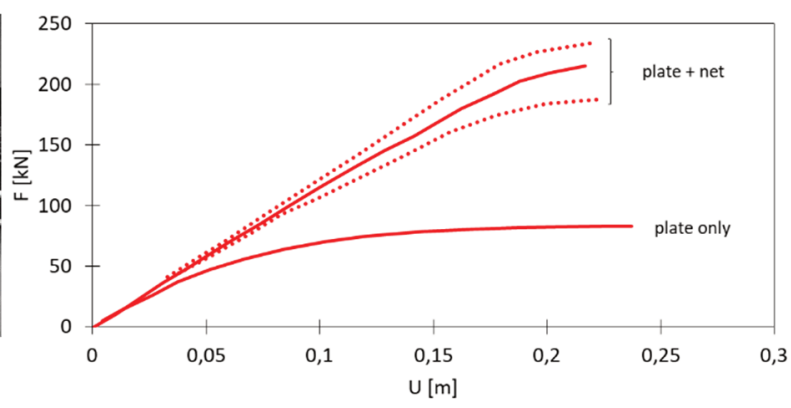

(b)

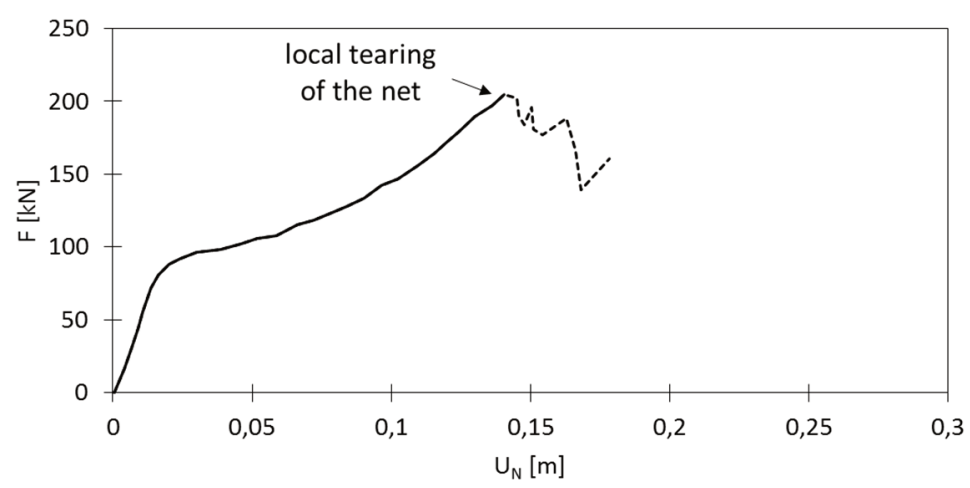

(c)

Figure 2 Example of punching test against soil: (a) Test setup; (b) Experimental results (from di Prisco et al. 2010) 
Table 1 Soil's geotechnical parameters

\begin{tabular}{lccc}
\hline Parameter & Symbol & Unit & Value \\
\hline Unit weight & $\gamma$ & $\mathrm{kN} / \mathrm{m}^{3}$ & 19 \\
Friction angle & $\phi^{\prime}$ & $\circ$ & 30 \\
Cohesion & $c^{\prime}$ & $\mathrm{kPa}$ & 10 \\
\hline
\end{tabular}

It is worth noting that, generally speaking, the characteristic curve is strictly dependent on all the dominant geometrical (plate dimension, anchor spacing, thickness of the unstable soil layer), and mechanical (net stiffness and strength, soil stiffness and strength) parameters of the problem. The application of the characteristic curve concept to a real design case would then require the calibration of a specific characteristic curve for each considered case. As briefly cited in section 1, the cited authors are, however, currently developing a simplified interpretative framework (based on a sub-structuring approach), capable of describing the main feature of the characteristic curve based on simple generalised variables, not necessarily requiring the run of extensive FEM numerical analyses or real experimental punching tests.

\section{$4 \quad$ Examples of application}

A test case representing a slightly cemented granular deposit with thickness $s=150 \mathrm{~cm}$ on steep sloping ground with inclination $\alpha=70^{\circ}$ and total height $H=30 \mathrm{~m}$ is schematically sketched in Figure 3a. The considered soil's geotechnical parameters are listed in Table 1. For the sake of simplicity, no watertable is considered, and an effective stress analysis is presented; furthermore, it is assumed that the steel mesh is installed along the whole slope, with a number $N_{a}=15$ of equally spaced anchors. The assumed design value of the global safety factor is $F_{S, d}=1.3$, and 10 equally spaced two-block type failure mechanisms have been considered. Figure $3 \mathrm{~b}$ shows the results of the aforementioned optimisation procedure, where, for each failure mechanism (labelled numbers refer to each slope failure mechanism), the required value of the pressure $q$ is maximised with respect to the inclination $\beta$ of the base of the toe block. The obtained maximum values of $q$ are then introduced into the characteristic curve (Figure 3c) in order to estimate the values of the corresponding normal displacement amplitude $U_{N}$ for each mechanism, and to derive a profile of the displacement envelope of the entire slope (Figure 4d).

Similar analysis can be easily run by varying, for example, the thickness of the unstable layer, as shown in Figures $3 e$ and $3 \mathrm{f}$, where the profiles of $U_{N}$ in the case $s=100 \mathrm{~cm}$ and $s=50 \mathrm{~cm}$, respectively, are plotted.

The effect of the thickness layer $s$ on the displacement envelope profiles is evident; in particular, a maximum displacement of about $12 \mathrm{~cm}$ is obtained at the toe of the slope in the case $s=150 \mathrm{~cm}$; which is remarkably reduced to $5 \mathrm{~cm}$ for $s=100 \mathrm{~cm}$. In the case $s=50 \mathrm{~cm}$, finally, the numerical analysis gives nil values for the horizontal displacement. This result can be interpreted as proof that the slope is stable with respect to the assigned value of $\mathrm{Fs}, \mathrm{d}(=1.3$, in this case), and no additional stabilising pressure is required by the steel mesh. Stability analyses for this reduced thickness of the granular layer, run without considering the presence of the anchored mesh, gave in fact safety factor values always larger than 1.6. 


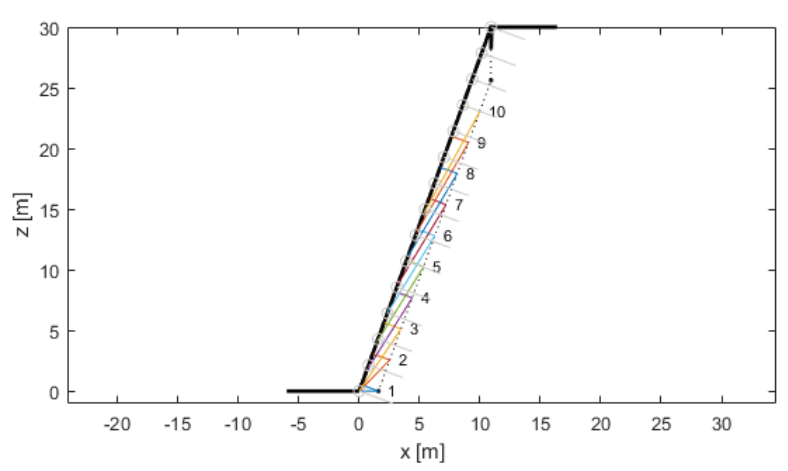

(a)

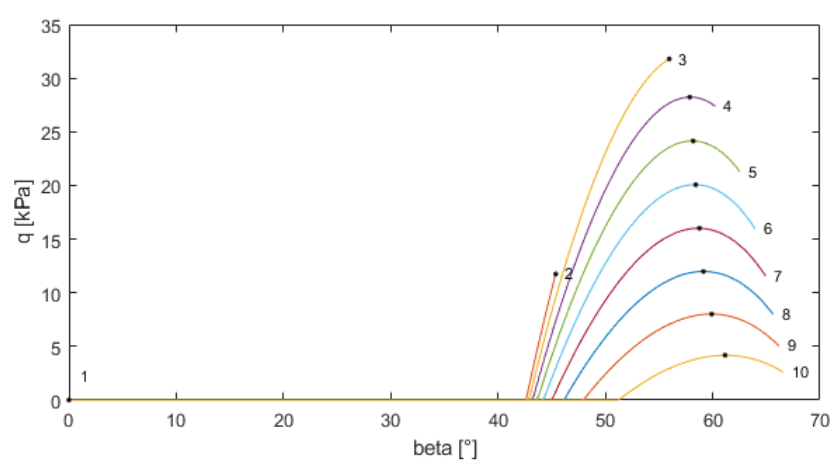

(b)

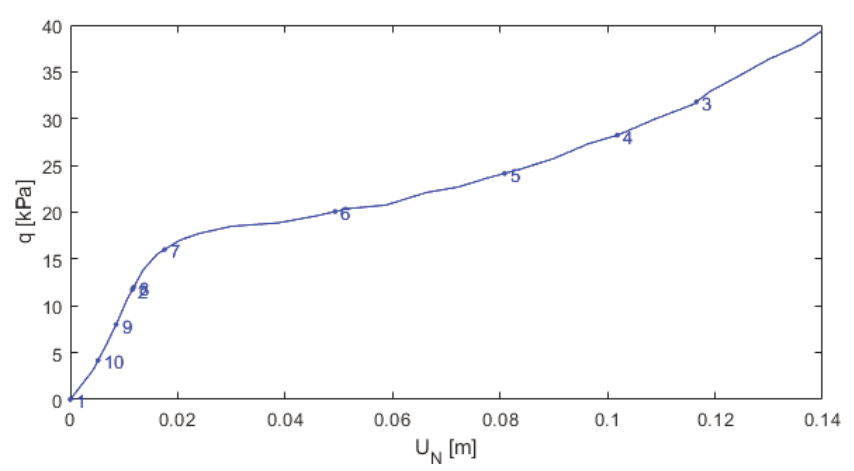

(c)

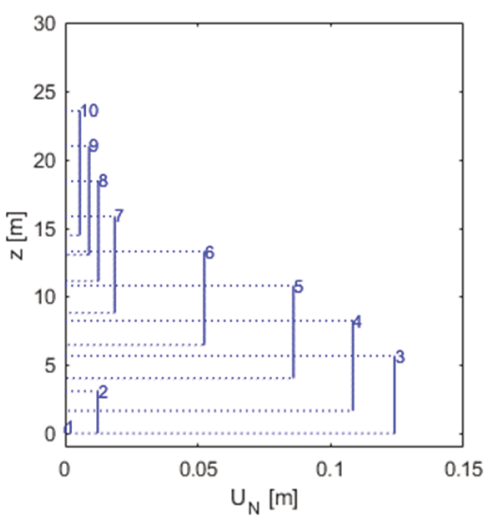

(d)

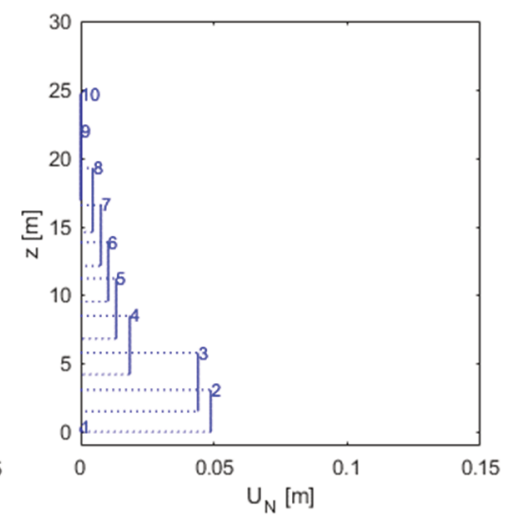

(e)

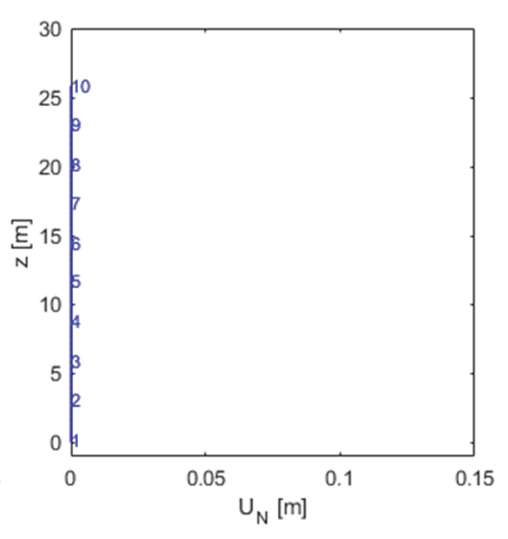

(f)

Figure 3 (a) Schematic view of the slope and of the 10 considered failure mechanisms; (b) Description of the optimisation procedure; (c) Estimation of the displacement through the characteristic curve; (d, e and f) Displacement envelope profiles along the slope for $s=150 \mathrm{~cm}, s=100 \mathrm{~cm}$ and $s=50 \mathrm{~cm}$, respectively 


\section{Discussion and conclusion}

In this paper, an innovative simplified design approach for anchored steel meshes on unstable soil layers on slopes has been presented. The procedure has been developed within the framework of the so called hybrid methods, developed for performing slope stability analyses by accounting for the presence of stabilising structures. In particular, the key concept is represented by the characteristic curve of the system, representing the relationship between the stabilising action and the slope movement. This allows, in particular, to directly relate the desired degree of safety of the slope (usually expressed by means of a global safety factor) to the corresponding slope displacement pattern, which is a measure of the performance of the stabilising system.

Some example numerical analyses (very fast to be run even on standard laptops), showed the efficiency of the methods in evaluating the slope displacement profile corresponding to the chosen value of the global safety factor of the slope, and proved the capability of the method to capture the main geometrical parameters affecting the problem.

From a design perspective, the proposed method is particularly suitable to perform extended parametrical analyses to rapidly optimise stabilising system both in terms of geometrical (e.g. number of anchors) and mechanical (e.g. type of steel mesh) properties.

\section{Acknowledgement}

This work would not have been possible without the work, the help and the support of Professor Claudio Di Prisco, Eng. Luca Flessati, and Eng. Katia Boschi.

\section{References}

Boschi, K, di Prisco, C, Flessati, L, Galli, A, Tomasin \& M 2019, 'Punching tests on deformable facing structures: numerical analyses and mechanical interpretation', in F Calvetti, F Cotecchia, A Galli \& C Jommi (eds), Proceedings of the 7th Italian National Congress of Geotechnical Researchers, Lecture Notes in Civil Engineering, vol. 40, pp. 429-437.

di Prisco, C, Besseghini, F \& Pisanò, F 2010, 'Modelling of the mechanical interaction between anchored wire meshes and granular soils', Geomechanics and Geoengineering: An International Journal, vol. 5, no. 3, pp. 137-152.

Galli, A \& Deana, M 2020, 'Hybrid design approaches for anchored wire meshes: towards a displacement based design' presented at the Transportation Research Board Annual Meeting 2020, Washington DC.

Galli, A \& di Prisco, C 2013, 'Displacement-based design procedure for slope-stabilizing piles', Canadian Geotechnical Journal, vol. 50, pp. 41-53.

Galli, A, Maiorano, RMS, Di Prisco, C \& Aversa, S 2017, 'Design of slope-stabilizing piles: from ultimate limit state approaches to displacement based methods', Rivista Italiana di Geotecnica, vol. 51, no. 3, pp. 77-93.

Italian Ministry of Education, University and Research 2011, Landslide Risk Mitigation using Sustainable Solutions, research program Rome. 
